\title{
The Relationship of Cognitive Impairment to Hypnotic Susceptibility in a Sample of Elderly: A Pilot Study
}

\author{
James R. Hall ${ }^{1,2^{*}}$, Melissa Pennington ${ }^{1}$, Erica Swicegood ${ }^{1}$, A. Scott Winter ${ }^{1}$ \\ ${ }^{1}$ Department of Psychiatry, University of North Texas Health Science Center at Fort Worth, Fort Worth, USA \\ ${ }^{2}$ Institute of Aging and Alzheimer's Disease Research, University of North Texas Health Science \\ Center at Fort Worth, Fort Worth, USA \\ Email: ${ }^{*}$ james.hall@unthsc.edu
}

Received October $6^{\text {th }}, 2011$; revised November $14^{\text {th }}, 2011$; accepted December $15^{\text {th }}, 2011$

\begin{abstract}
The relationship between cognitive impairment and hypnotic susceptibility in elderly individuals was investigated. The Stanford Hypnotic Suggestibility Scale (SHSS; Form C Modified) was administered to 30 volunteers between the ages of $65-87$ who had been evaluated for cognitive impairment. The sample consisted of 14 normal controls, 8 subjects with mild Alzheimer's disease, and 8 subjects with mild cognitive impairment. No significant differences between groups were found on performance on the SHSS. Results suggest that that hypnosis may be a useful adjunct to enhance the effectiveness of therapeutic interventions for elderly patients with mild dementia.
\end{abstract}

Keywords: Hypnotic Suggestibility; Normal Aging; Alzheimer's Disease; Mild Cognitive Impairment

\section{Introduction}

As the population ages and requires higher levels of medical care including surgery and invasive procedures, there is an increased need for adjunctive approaches that don't have the negative side-effects of many medications, analgesics, and anesthesia. Hypnosis is a viable non-pharmacological intervention with few if any negative side effects. Although there have been a few controlled trials studying hypnosis with the elderly, there is evidence supporting the utility of hypnosis with older adults for a number of medical conditions and treatments. Beneficial effects for the use of hypnosis with older adults have been reported for the control of chronic pain (Morone \& Greco, 2007), the control of osteoarthritis pain (Gay, Philippot, \& Luminet, 2002), the treatment of obesity (Cherniack, 2008), the use during invasive medical procedures (Lutgendorf, et al., 2007), and as an adjunct during colonoscopy (Elkins, et al., 2006).

A number of issues have been raised regarding the use of hypnosis with the elderly. It has been argued that older adults are not as capable of being hypnotized as younger people (Luskin, et al., 2000). The effectiveness of hypnosis has been related to the hypnotizability of the subject (Hilgard, 1965; Braffman \& Kirsch, 1999; Montgomery, DuHamel, \& Redd, 2000; Milling, Coursen, Shores, \& Waszkiewicz, 2010). Research on age and hypnotizability has suggested that hypnotic susceptibility is a trait that does not vary significantly as individuals age (Piccone, Hilgard, \& Zimbardo, 1989). Page and Green (2007) found that hypnotic suggestibility scores decrease from age 17 - 40 and then tend to increase and stabilize as a person ages. Lutgendorf, et al. found that increasing age did not affect hypnotizability nor diminish the utility of hypnosis during invasive medical procedures. Taken together these findings indicate that aging is not a significant determinant of hypnotizability.

${ }^{*}$ Corresponding author.
In addition to the concern with age as a factor in hypnotizability there are questions about the use of hypnosis with elderly who have cognitive impairment. The hypnotic process has been described as "attentive receptive concentration" (Spiegel \& Spiegel, 1987) that requires intact cognitive functioning. Recent neurophysiological evidence supports the importance of attentional mechanisms in hypnotizability (Raz, Fan, \& Posner, 2006). The anterior cingulate and right frontal and parietal areas (Faymonville, Boly, \& Laureys, 2006) are areas posited to be involved in the attentional network. Dementia, especially Alzheimer's disease (AD), is marked by deficits in the functioning of these areas. This raises questions about the utility of hypnosis in seniors who may have neurocognitive deficits. Another type of cognitive impairment found in the elderly, Mild Cognitive Impairment (MCI), is well-established as a precursor to dementia (Petersen, 2004) and is seen as a neuropathological progression between normal aging and patients with Alzheimer's disease (AD) (Braak \& Braak, 1995). A recent study (Redel, et al., 2010) found that selective attention, which was related to fronto-parietal networks, was significantly reduced in MCI patients and further impaired in AD. This neurophysiological evidence suggests that individuals with even mild cognitive deficits may have impaired attentional processes that may limit their ability to be hypnotized.

To our knowledge, no studies have looked specifically at the relationship between the level of cognitive functioning and hypnotic susceptibility in the elderly. The present study investigates hypnotic susceptibility in individuals with mild Alzheimer's disease, mild cognitive impairment and cognitively normal elderly. Understanding these associations may have an impact on the clinical use of hypnosis with elderly individuals with cognitive impairment.

\section{Method}

Setting: The study was conducted at a large urban health sci- 
ence center. The sample for the study was drawn from individuals who had attended a university based geriatric medicine clinic and had been assessed as having either normal cognition, mild cognitive impairment, or mild dementia by consensus diagnosis. This evaluation was based on clinical diagnoses and neurocognitive testing. Individuals scoring within the normal range on a standard battery of neuropsychological tests including tests of memory (WMS-III) and executive functions (Clock Drawing and Trail Making Tests A \& B) were classified as normal. Individuals were diagnosed with Mild Cognitive Impairment $(\mathrm{MCI})$ if they scored within the impaired range $(1.5 \mathrm{sd}$ below norms for age and education) on at least one neurocognitive test of the standard battery and had subjective reports of cognitive change without the presence of significant impairment in everyday functioning. Individuals were diagnosed with dementia if they met DSM-IV criteria for dementia. The final pool of possible participants was composed of 120 individuals who had attended the clinic within the past 12 months and had been diagnosed as cognitively intact, MCI or having dementia and had a Clinical Dementia Rating (CDR) global score of $0, .5$ or 1.0 (Morris, 1997). The CDR is a global assessment instrument that yields a score between 0 and 3 that is regularly used in clinical and research settings to stage dementia severity (Morris, 1997).These individuals (58 normal; 20 MCI and 42 with a dementia diagnosis) were contacted by a member of the research team and asked if they were interested in participating in research on hypnosis. The nature of the study was completely explained to each person and those agreeing to participate were consented and seen in the geriatric psychiatry clinic.

Participants: The final sample consisted of 30 individuals: 14 normal controls (NC), 8 with mild cognitive impairment (MCI), and 8 with mild dementia. The NC group was composed of 14 volunteers without a history of cognitive impairment and who had been clinically assessed as cognitively intact during the past year. To determine if change in cognitive functioning had occurred in the Normal Controls since the initial evaluation, the Mini-Mental Status Exam (MMSE) (Folstein, Folstein, McHugh, \& Fanjiang, 2001) was administered at the time of their appointment. The MMSE is one of the most widely used brief screening instruments of general cognitive functioning and provides a total score. Those scoring within normal range for age and education were assigned to the Normal Control group. The MCI group consisted of 8 individuals diagnosed with Mild Cognitive Impairment and had a CDR score of $\leq 0.5$. The Mild Dementia group consisted of 7 individuals diagnosed with Alzheimer's disease and one diagnosed with a mixed Alzheimer's and Vascular Dementia who had a global CDR score of $\leq 1.0$.

Procedures: All individuals who consented to participate were seen in single individual appointments lasting between 60 and 90 minutes. Participants were administered the Stanford Hypnotic Suggestibility Scale (SHSS Form C Modifed). The hypnotic suggestions included in the study (see Table 1.) were those that have been shown to be non-duplicative and excluded the mosquito hallucination and the anosmia hallucination (Naring, Hoogduin, \& Keijser, 2004). Standard procedures were followed although all items were administered to every subject. The SHSS-C was administered by two senior psychiatry residents (authors M.P \& E.S.) who had received extensive training in hypnosis and the administration of the scale. The study was approved by the Institutional Review Committee and all participants provided written informed consent. Patients in the
Table 1.

Hypnotic suggestions.

\begin{tabular}{cc}
\hline Hand lowering & Age regression \\
Moving hands apart & Arm immobilization \\
Taste hallucination & Auditory hallucination \\
Arm Rigidity & Negative visual hallucination \\
Dream & Posthypnotic amnesia \\
\hline
\end{tabular}

Mild Cognitive Impairment and Mild Dementia group were determined from interview and neuropsychological testing to have the capacity to understand the written information in the consent form and to have the capacity to give informed consent.

Analysis: Chi-Square was used to assess differences on categorical data and ANOVA were conducted to determine differences between the groups on continuous variables. Level of significance was set at $p \leq .05$ for all analyses.

\section{Results}

There were 11 males and 19 females in our sample with a mean age of 74.30 years and an average of 13.93 years of education. Due to the small sample size the power of the study was low and the likelihood of making a Type II error was increased. The characteristics of the sample and score on the Stanford Hypnotic Suggestibility Scale are presented in Table 2. The groups did not differ on gender (Chi-square $(\mathrm{df}=1)=2.133, p$ $=.144)$; age $(\mathrm{F}=.972,2.27), p=.391)$ or education $(\mathrm{F}=.462$, (df 2.27) $p=.635$ ). ANOVA revealed no significant differences between the diagnostic groups on total score $(\mathrm{F}=1.642(\mathrm{df}$ 2/27) $p=.212$ ).

\section{Discussion}

The current study found that there were no significant differences on hypnotic susceptibility as measured by the Stanford Hypnotic Suggestibility Scale between normal elderly, elderly with Mild Cognitive Impairment or elderly with Mild Dementia. While other studies have evaluated the link between aging and hypnotic susceptibility, this is the first study to separate participants by level of cognitive functioning. The results of the current need to be interpreted with caution due to the small sample size which increases the likelihood of making a Type II error. With this caveat the results of the current study can be seen as offering initial support for hypnosis as a useful adjunct to enhance the effectiveness of therapeutic interventions for elderly patients with mild dementia. If further research supports the use of hypnosis with this population then hypnosis has the potential for treating a number of disorders without the risks related to medication use in the geriatric population.

Our findings suggest that at least in our sample, individuals with relatively mild cognitive impairment (MCI and mild AD) retain adequate attentional processing ability to allow the application of hypnosis. The SHSS has no norms specifically for the elderly or elderly with cognitive impairments. Without comparative norms it is difficult to evaluate the relationship between the scores on SHSS and expected response to hypnosis in this population. Exploratory post hoc analysis of the items found that suggestions for auditory and visual hallucinations were unsuccessful across all groups. This may indicate that 
Table 2.

Mean and standard deviation of age, education, and stanford hypnotic suggestibility scale-Form C (SHSS-C) total score.

\begin{tabular}{cccc}
\hline Mean (SD) & Normal Controls N $=14$ & Mild Cognitive Impairment N $=8$ & Mild Dementia N $=8$ \\
\hline Age & $74.21(6.55)$ & $72.00(7.13)$ & $76.75(6.99)$ \\
Education & $14.21(1.97)$ & $14.00(2.20)$ & $13.93(1.77)$ \\
SHSS C Total Score & $4.53(1.90)$ & $3.81(3.55)$ & $5.30(1.58)$ \\
\hline
\end{tabular}

response to these suggestions is rare in older individuals and that they these items may have little value in assessing susceptibility in the elderly. There are a number of limitations that impact the generalizability of our findings. Most notable is the small size and homogeneity of our sample. All the patients were Caucasian and attended the same clinic. The sample was middle class and relatively well educated. Five of the participants were referred by psychiatrists, and as such carried diagnoses such as Major Depressive Disorder or Generalized Anxiety Disorder, which could arguably affect attention and concentration and thus could skew results although there were no differences between these participants and others on total score.

This pilot study opens the door for future studies involving a larger sample size of patients with different ethnic origins, educational backgrounds, and absence of psychiatric diagnoses. The current research can not speak to the specific uses of hypnosis with the mildly impaired elderly but the findings suggest that hypnosis may be a useful tool even with elderly who have been diagnosed with mild levels of cognitive impairment.

\section{REFERENCES}

Braak, H., \& Braak, E. (1995). Staging of Alzheimer's disease-related neurofibrillary changes. Neurobiology of Aging, 16, 271-278. doi:10.1016/0197-4580(95)00021-6

Braffman, W., \& Kirsch, I. (1999). Imaginative suggestibility and hypnotizability: An empirical analysis. Journal of Personality and Social Psychology, 77, 578-587. doi:10.1037/0022-3514.77.3.578

Cherniack, E. P. (2008). Potential applications for alternative medicine to treat obesity in an aging population. Alternative Medicine Review, $13,34-42$.

Elkins, G., White, J., Patel, P., Marcus, J., Perfect, M. M., \& Montgomery, G. H. (2006). Hypnosis to manage anxiety and pain associated with colonoscopy for colorectal cancer screening: Case studies and possible benefits. International Journal of Clinical and Experimental Hypnosis, 54, 416-431. doi:10.1080/00207140600856780

Faymonville, M. E., Boly, M., \& Laureys, S. (2006). Functional neuroanatomy of the hypnotic state. Journal of Physiology, Paris, 99, 483-491. doi:10.1016/j.jphysparis.2006.03.018

Folstein, M. F., Folstein, S. E., McHugh, P. R., \& Fanjiang, G. (2001). Mini-mental state examination user's guide. Odessa, FL: Psychological Assessment Resources.

Gay, M. C., Philippot, P., \& Luminet, O. (2002). Differential effectiveness of psychological interventions for reducing osteoarthritis pain. European Journal of Pain, 6, 1-16. doi:10.1053/eujp.2001.0263

Hilgard, E. R. (1965). Hypnosis. Annual Review of Psychology, 16,
157-180. doi:10.1146/annurev.ps.16.020165.001105

Luskin, F. M., Newell, K. A., Griffith, M., Holmes, M., Telles, S., DiNucci, E., et al. (2000). A review of mind/body therapies in the treatment of musculoskeletal disorders with implications for the elderly. Alternative Therapies in Health and Medicine, 6, 46-56.

Lutgendorf, S., Lang, E. V., Berbaum, K. S., Russell, D., Berbaum, M. L., Logan, H., et al. (2007). Effects of age on responsiveness to adjunct hypnotic analgesia during invasive medical procedures. Psychosomatic Medicine, 69, 191-199. doi:10.1097/PSY.0b013e31803133ea

Milling, L. S., Coursen, E. L., Shores, J. S., \& Waszkiewicz, J. A. (2010). The predictive utility of hypnotizability: The change in suggestibility produced by hypnosis. Journal of Consulting and Clinical Psychology, 78, 126-130. doi:10.1037/a0017388

Montgomery, G. H., DuHamel, K. N., \& Redd, W. H. (2000). A metaanalysis of hypnotically induced analgesia: How effective is hypnosis? International Journal of Clinical and Experimental Hypnosis, 48, 138-153. doi: $10.1080 / 00207140008410045$

Morone, N. E. \& Greco, C. M. (2007). Mind-body interventions for chronic pain in older adults: A structured review. Pain Medicine, 8, 359-375. doi:10.1111/j.1526-4637.2007.00312.x

Morris, J. C. (1997). Clinical dementia rating: A reliable and valid diagnostic and staging measure for dementia of the Alzheimer type. International Psychogeriatrics, 9, 173-176. doi: $10.1017 /$ S1041610297004870

Naring, G. W., Hoogduin, K. A., \& Keijser, C. M. (2004). A rasch analysis of the Stanford hypnotic susceptibility scale. International Journal of Clinical and Experimental Hypnosis, 52, 250-259. doi: $10.1080 / 0020714049052350$

Page, R. A., \& Green, J. P. (2007). An update on age, hypnotic suggestibility, and gender: A brief Report. American Journal of Clinical Hypnosis, 49, 283-287.

Petersen, R. C. (2004). Mild cognitive impairment as a diagnostic entity. Journal of Internal Medicine, 256, 183-196. doi:10.1111/j.1365-2796.2004.01388.x

Piccione, C., Hilgard E., \& Zimbardo P. (1989). On the degree of stability of measured hypnotizability over a 25 -year period. Journal of Personality and Social Psychology, 56, 289-295. doi: 10.1037/0022-3514.56.2.289

Raz, A., Fan, J., \& Posner, M. (2006). Neuroimaging and genetic associations of attentional and hypnotic processes. Journal of Physiology, Paris, 99, 483-491. doi:10.1016/j.jphysparis.2006.03.003

Redel, P., Bublak, P., Sorg, C., Kurz, A., Forstl, H., Muller, H. J., et al. (2010). Deficits of spatial and task-related attentional selection in mild cognitive impairment and Alzheimer's disease, Neurobiology of Aging. [Epub ahead of print].

Spiegel, H., \& Spiegel, D. (1987). Trance and treatment: Clinical uses of hypnosis. Washington DC: American Psychiatric Press. 